\title{
Changes in aortic arch geometry and the risk for Stanford B dissection
}

\author{
Peng Qiu ${ }^{1 \#}$, Junchao Liu ${ }^{1 \#}$, Yuqian Chen ${ }^{2}$, Binshan $\mathrm{Zha}^{3}$, Kaichuang $\mathrm{Ye}^{1}$, Jinbao Qin ${ }^{1}$, Peipei Hao ${ }^{4}$, \\ Jiwen Kang ${ }^{5}$, Chao Zhang ${ }^{6}$, Huagang $\mathrm{Zhu}^{3}$, Xinwu $\mathrm{Lu}^{1}$
}

${ }^{1}$ Department of Vascular Surgery, Shanghai Ninth People's Hospital Affiliated to Shanghai Jiao Tong University School of Medicine, Shanghai, China; ${ }^{2}$ School of Public Economics and Administration, Shanghai University of Finance and Economics, Shanghai, China; ${ }^{3}$ Department of Vascular and Thyroid Surgery, Department of General Surgery, The First Affiliated Hospital of Anhui Medical University, Hefei, China; ${ }^{4}$ Department of Radiology, the Affiliated Tongji Hospital of Tongji University, Shanghai, China; ${ }^{5}$ Department of Radiology, Shanghai Jiading Kangqiao Hospital, Shanghai, China; ${ }^{6}$ Department of Radiology, Yijishan Hospital Affiliated to Wannan Medical College, Wuhu, China

Contributions: (I) Conception and design: P Qiu, J Liu, X Lu, H Zhu; (II) Administrative support: K Ye, J Qin, B Zha; (III) Provision of study materials or patients: P Qiu, J Liu, P Hao, J Kang, C Zhang, Y Chen; (IV) Collection and assembly of data: P Qiu, J Liu, P Hao, J Kang, C Zhang; (V) Data analysis and interpretation: P Qiu, Y Chen; (VI) Manuscript writing: All authors; (VII) Final approval of manuscript: All authors.

"These authors contributed equally to this work.

Correspondence to: Huagang Zhu, MD, PhD or Xinwu Lu, MD, PhD. Department of Vascular and Thyroid Surgery, Department of General Surgery, First Affiliated Hospital of Anhui Medical University, or Department of Vascular Surgery, Shanghai Ninth People's Hospital Affiliated to Shanghai Jiao Tong University School of Medicine, No. 218 Jixi Road, Hefei 230000, China or No. 639 Zhizaoju Road, Shanghai 200000, China.

Email: huagzhu@yeah.net or luxinwu@shsmu.edu.cn.

Background: The increase in aortic diameter is not closely associated with type B aortic dissection (TBAD); morphological risk factors other than aortic diameter may help to better identify patients at risk for TBAD. The purpose of this study was to investigate possible morphological factors associated with the occurrence of TBAD.

Methods: This study was a retrospective, multicenter, cross-sectional study. We collected 94 patients with TBAD as the TBAD group and 534 patients with healthy aortas as the healthy control group. Morphometric data were collected on three-dimensional models of the thoracic aorta. A propensity score matching (PSM) analysis was conducted to reduce the potential for confounding by baseline factors.

Results: The number of patients in the TBAD group was 75 after PSM. Longer lengths of the aortic arch $(28.00 \pm 7.42$ vs. $25.14 \pm 7.11 \mathrm{~cm})$ were observed in patients with TBAD. The width $(80.04 \pm 17.27$ vs. $71.73 \pm 15.55 \mathrm{~mm})$ and height $(24.92 \pm 11.39$ vs. $19.37 \pm 10.10 \mathrm{~mm})$ of the aortic arch in patients with TBAD were both larger than those of healthy controls. The morphological changes associated with the occurrence of type B acute dissection were most pronounced in the geometry of the aortic arch.

Conclusions: This study demonstrates that TBAD was associated with longer lengths of aortic arch and with larger arch height and width.

Keywords: Aortic arch; anatomy; aortic dissection

Submitted Apr 11, 2020. Accepted for publication Oct 13, 2020.

doi: $10.21037 /$ jtd-20-1643

View this article at: http://dx.doi.org/10.21037/jtd-20-1643 


\section{Introduction}

Aortic dissection is the most common fatal aortic disease. Despite the development of diagnostic strategies, endograft design, and surgical treatment for aortic dissection in the last 20 years, the in-hospital mortality rate of patients presenting with Type B aortic dissection (TBAD) has remained at 13\% (1). For this fatal aortic disease, the benefits of treatment for stable and asymptomatic but high-risk conditions are far better than treatment after the presentation of acute and catastrophic symptoms (2). Therefore, identification of the risk factors for TBAD and prevention before an occurrence of acute and catastrophic symptoms are the best ways to reduce the mortality and treatment costs of TBAD (3). Hypertension, increasing age, atherosclerosis, and genetic predisposition are well-established risk factors for TBAD, but it is difficult to improve cost-effectiveness in screening programs by focusing on high-risk patients using these common risk factors. Numerical and experimental evidence has illustrated that morphological changes in the aorta can affect the mechanical stresses $(4,5)$, which are closely related to the morphological features of endothelial cells (6). Current guidelines recommend that patients with an ascending aortic transverse diameter exceeding $5.5 \mathrm{~cm}$ undergo prophylactic treatment to prevent type A aortic dissection, but for TBAD, there are no such appropriate morphological risk factors (2). Therefore, the identification of morphological risk factors may help to better identify patients at risk for TBAD.

Our study considered the following points to identify the potential morphological risk factors for TBAD. First, the aortic dilation is occurring not only transversally but also longitudinally. Although there was no correlation between aortic diameter and TBAD, aortic elongation has been shown to be associated with TBAD. Second, in addition to the length of the aorta, other morphological indicators, such as the geometry of the aortic arch, may indicate the predisease aortic morphology, which has not been previously studied in TBAD. Third, considering the difficulty of acquiring imaging prior to dissection onset, we intended to identify some morphological indicators that remained relatively stable before and after the onset of TBAD to describe the pre-disease aortic morphology. Therefore, we retrospectively collected computed tomography (CT) angiography data from TBAD patients and healthy control subjects and compared the morphology parameters (the length of each segment of the aorta and the geometry of the aortic arch) between the two groups. Fourth, it is widely accepted that advancing age, gender, body surface area (BSA), and comorbidities might have an effect on the aortic morphologies (7-9), but most previous studies failed to compare well-matched patient groups, allowing selection bias to influence the results (10-12). In this study, aiming to determine the association between the occurrence of TBAD and aortic morphology, we intended to use propensity score matching (PSM) to control for selection bias. We present the following article in accordance with the STROBE reporting checklist (available at http://dx.doi.org/10.21037/ jtd-20-1643).

\section{Methods}

\section{Study population}

This study was a retrospective, multicenter, cross-sectional study. Patients in the TBAD group were diagnosed with acute Stanford TBAD at two institutions (Shanghai Ninth People's Hospital Affiliated to Shanghai Jiao Tong University School of Medicine and Department of Vascular and The First Affiliated Hospital of Anhui Medical University). We retrospectively evaluated CT scans of consecutive TBAD patients who underwent thin-cut (0.6- $\mathrm{mm})$ contrast-enhanced CT angiography between January 2017 and December 2018. The exclusion criteria for the TBAD group were as follows: (I) patients with connective tissue disease (Marfan's disease, LoeysDietz syndrome or Ehlers-Danlos syndrome); (II) iatrogenic dissection; (III) patients with other aortic diseases such as aneurysms; (IV) patients with previous aortic surgery; $(\mathrm{V})$ patients with previous cardiothoracic disease or cardiothoracic surgery; (VI) patients with diseases that might cause distortion of the thoracic aortic morphology (pulmonary nodules with a diameter $>3 \mathrm{~cm}$, mediastinal masses or lymph nodes with a diameter $>1 \mathrm{~cm}$, pneumothorax, pulmonary bullae with a diameter $>3 \mathrm{~cm}$, history of thoracic and mediastinal surgery, etc.); and (VII) patients with diseases that might cause distortion of the shape of the thoracic wall (scoliosis, barrel chest, pectus carinatum, history of spinal surgery, etc.). Missing data in the database has been excluded.

We retrospectively evaluated CT scans of consecutive patients with healthy aortas who underwent thin-cut (0.6- $\mathrm{mm})$ contrast-enhanced CT angiography or contrastenhanced chest CT at one institution (Shanghai Ninth People's Hospital Affiliated to Shanghai Jiao Tong University School of Medicine) between April 2018 and 

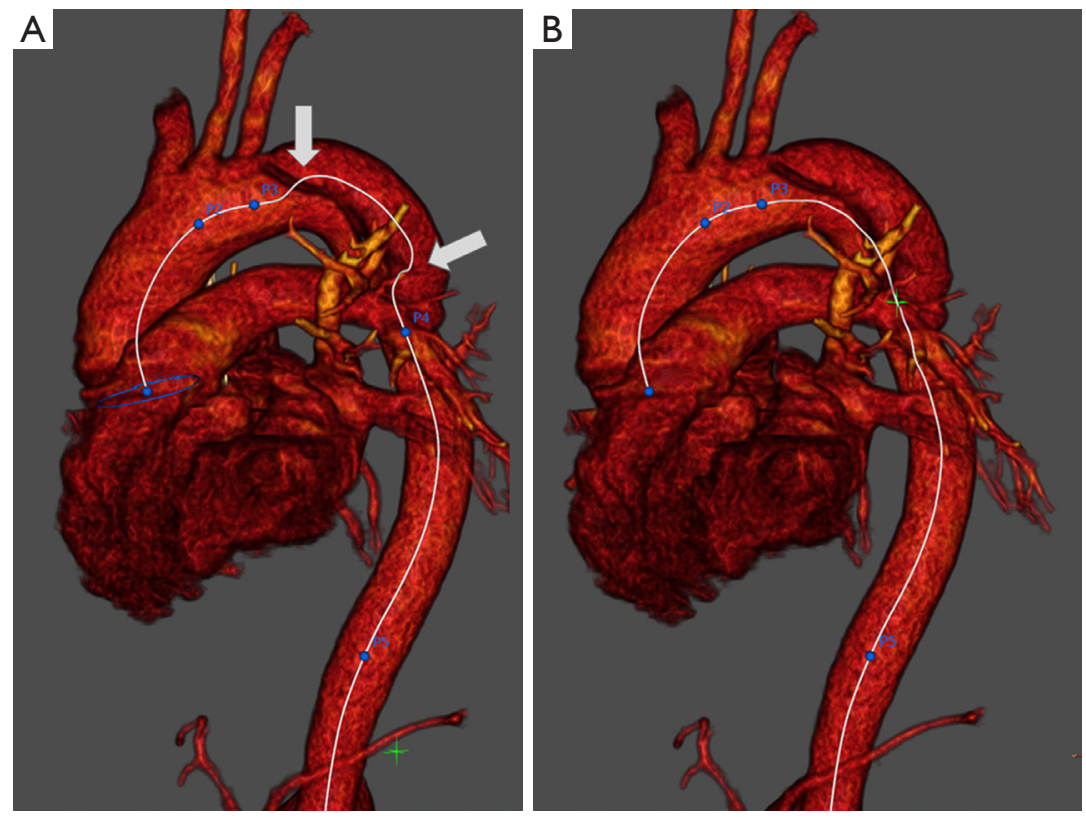

Figure 1 A center line was placed manually from the center of false lumen (A) to the center of total artery lumen (B), the arrows indicate the position of the center line was manually replaced.

December 2018 as a healthy control group. The exclusion criteria for the healthy control group were as follows: (I) patients with suspected or known aortic disease; (II) patients with previous cardiothoracic disease or cardiothoracic surgery; (III) patients with diseases that might cause distortion of the thoracic aortic morphology; and (IV) patients with diseases that might cause distortion of the shape of the thoracic wall. Missing data in the database has been excluded.

The procedures followed were in accordance with the Declaration of Helsinki (as revised in 2013), and the study protocol was reviewed and approved by the local ethics committee (SH9H-2019-T144-2). The need for written patient consent was waived because of the observational nature of this study. This retrospective factorial study was registered with the Chinese Clinical Trial Registry, and its registration number is ChiCTR2000029219.

\section{Image analysis}

Digital Imaging and Communications in Medicine (DICOM) standard data from eligible patients were transferred for further analysis in an anonymized fashion. Threedimensional multiplanar reconstruction was performed with EndoSize software version 3.1 (Rennes, France).

A center line was created from the sinotubular junction to the diaphragmatic level of the descending thoracic aorta.
In the presence of $\mathrm{AD}$, we manually place the seed points of the centerline at the center of the total artery lumen (i.e., both the true and false lumens) to obtain the adjusted predissected aortic centerline (Figure 1). According to previous study $(7,12)$, the thoracic aorta was divided into 4 segments by appropriate planes perpendicular to the center line (Figure 2): 1. ascending aorta (from the sinotubular junction to the origin of the brachiocephalic artery); 2. aortic arch (from the origin of the brachiocephalic artery to the origin of the left subclavian artery); 3. proximal descending thoracic aorta (from the origin of the left subclavian artery to the level of the pulmonary trunk bifurcation); and 4 . distal descending thoracic aorta (from the level of the pulmonary trunk bifurcation to the diaphragm). The length of each segment was measured as the center line distance between the previously defined points.

Aortic arch parameters were measured in the aortic view. The arch width was defined as the maximum distance between the outer curvature of the ascending aorta and the descending aorta. The arch height was defined as the vertical distance between the origin of the brachiocephalic artery and the arch vertex. The arch angle was measured as the angle between the line between the origin of the brachiocephalic artery and the origin of the left subclavian artery and the horizontal line.

According to the data of previous studies, the length of 


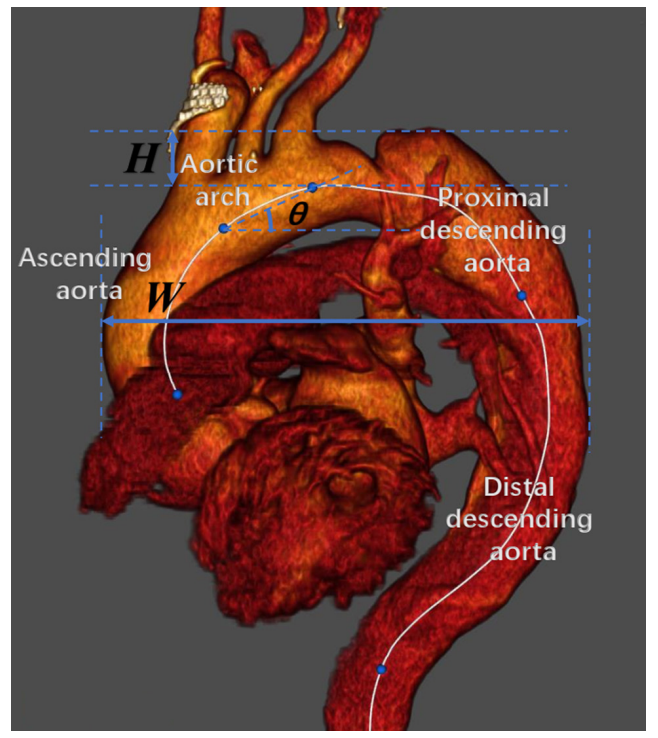

Figure 2 Aortic view of thoracic aorta illustrating aortic measurements. The thoracic aorta was divided into 4 segments: ascending aorta, aortic arch, proximal descending thoracic aorta, and distal descending thoracic aorta. The arch width $(W)$ was defined as the maximum distance between the outer curvature of the ascending aorta and the descending aorta. The arch height $(H)$ was defined as the vertical distance between the origin of the brachiocephalic artery and the arch vertex. The arch angle $(\theta)$ was measured as the angle between the line between the origin of the brachiocephalic artery and the origin of the left subclavian artery and the horizontal line.

healthy aortic arch was estimated to be $30 \pm 10 \mathrm{~mm}$, and the length of dissected aortic arch to be $35 \mathrm{~mm}$. We accepted a $\mathrm{P}<0.05$ as acceptable and a study with $80 \%$ power. With the enrollment ratio of 1 , the sample size of the experimental group and the control group was estimated to be 63:63, respectively. We estimated that only $80 \%$ of the patients in the TBAD group were included in the final comparison after PSM. Therefore, we finally estimated that the TBAD group needed at least 78 cases, and the healthy control group needed more than 78 cases, and the more cases, the better.

We used Heuts's retrospective modeling method to obtain the adjusted predissection dimension (12-14). Rylski et al. revealed that the length of the descending aorta increased by $3 \%$ on average after the occurrence of dissection, while the lengths of the ascending aorta and the aortic arch did not increase (14). As in their study, we modeled the aortas of TBAD patients to pre-dissection length by deducting $3 \%$ of the measured length of the descending aorta.

\section{Statistical analysis}

The distribution of continuous variables was assessed for normality by the Shapiro-Wilk test and was assessed visually by inspection of histograms and standardized normal probability (P-P) plots. Continuous variables, expressed as the mean \pm standard deviation, were compared using the independent $t$-test. Categorical variables, expressed as frequencies and percentages, were compared using Pearson's $\chi^{2}$ test. PSM was used to reduce the potential for confounding by baseline factors (15). Patients in the healthy control group were matched 1-to-1 with patients in the TBAD group, with nearest-neighbor matching without replacement and a matching tolerance (caliper) of 0.2 . During matching, maximization of execution performance and randomization of case order were performed. Standardized differences for each variable and for each treatment group were calculated to assess the balance, with values $<0.10$ indicating a good balance. All data were analyzed using SPSS statistical software version 24.0 (Chicago, $\mathrm{IL}$ ). $\mathrm{P}<0.05$ was considered to be statistically significant.

\section{Results}

\section{Patient characteristics}

A total of 94 patients were included in the TBAD group, and 534 subjects were included in the healthy control group. Participation at each stage is shown in a flow diagram (Figure 3). Patients in the TBAD group were younger than patients in the healthy control group $(57.46 \pm 12.00 v s .65 .24 \pm 12.21$ years, $\mathrm{P}<0.001)$. There were more men in the TBAD group than in the healthy control group $(80.9 \%$ vs. $58.4 \%, \mathrm{P}<0.001)$. Patients in the TBAD group weighed more $(70.24 \pm 9.36$ vs. $63.27 \pm 11.00 \mathrm{~kg}, \mathrm{P}<0.001)$, had a higher BSA $(1.75 \pm 0.14 v s$. $\left.1.66 \pm 0.17 \mathrm{~m}^{2}, \mathrm{P}<0.001\right)$, and more frequently had hypertension (86.2\% vs. $37.1 \%, \mathrm{P}<0.001)$ and diabetes mellites $(28.7 \%$ vs. $15.7 \%, \mathrm{P}=0.002)$. The comparison of baseline characteristics between the two groups before PSM is summarized in Table 1. Missing data in the database has been excluded.

\section{Propensity score matching}

We performed PSM to reduce the potential for confounding by baseline factors (gender, age, BSA, rate of hypertension, 


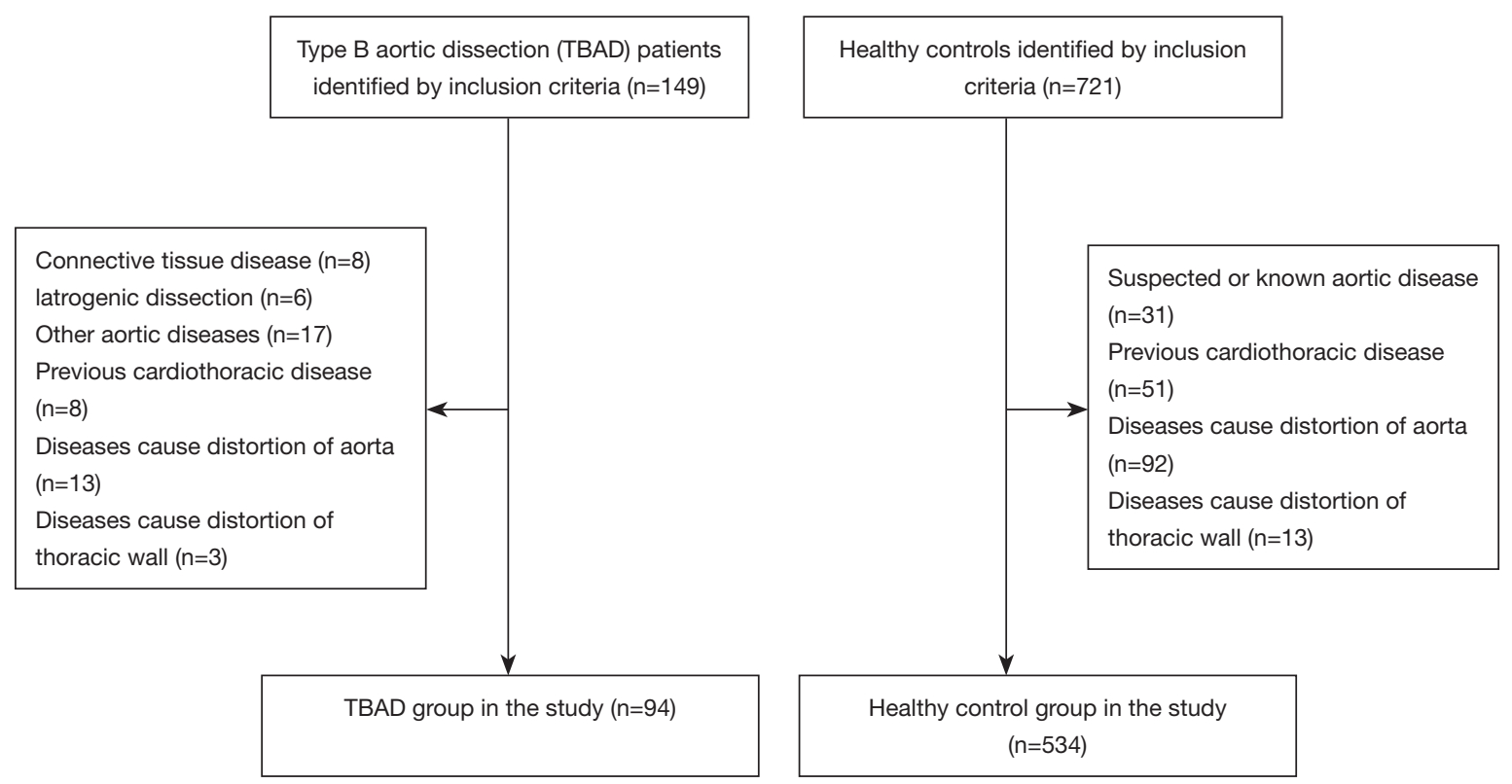

Figure 3 Flow diagram of participation.

Table 1 The comparison of baseline characteristics between the TBAD group and Healthy controls group

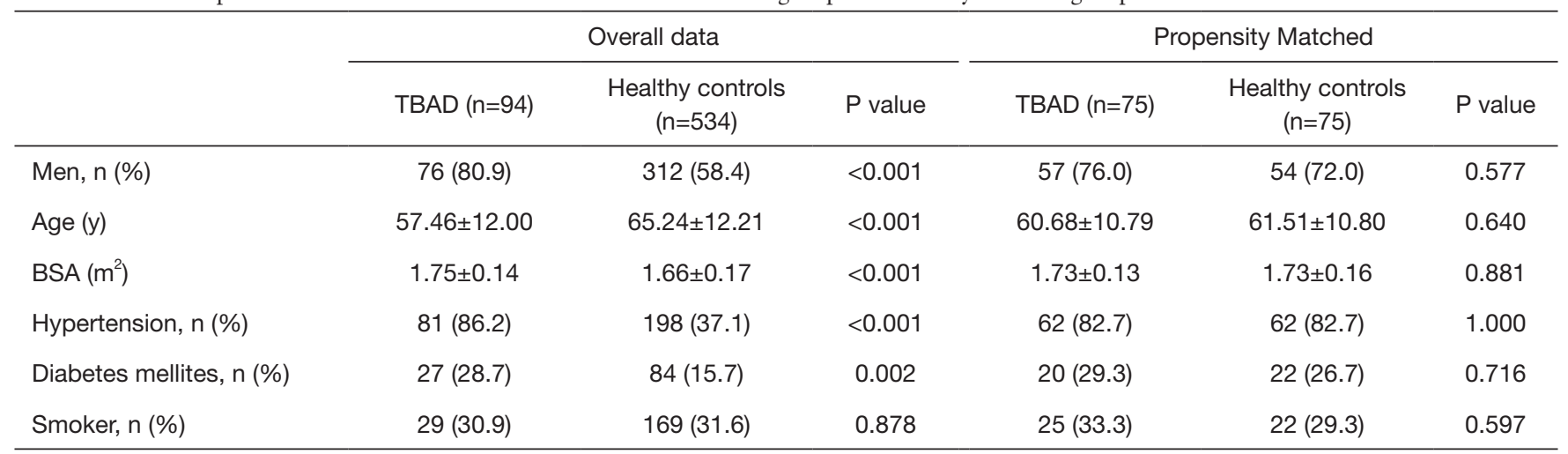

TBAD, type B aortic dissection; BSA, body surface area.

rate of diabetes mellites, and rate of smoking). Scoring performed well for balancing confounders. Before matching, there were 6 statistically significant differences between the TBAD group and the healthy control group. In the course of PSM, we successfully matched 75 TBAD patients to 75 healthy control subjects. The matched pairs did not differ with regard to any baseline characteristics (Table 1).

\section{Aortic geometry}

We observed 12 geometric indicators of the aorta. In the PSM cohort, we observed longer lengths of the aortic arch $(28.00 \pm 7.42$ vs. $25.14 \pm 7.11 \mathrm{~mm}, \mathrm{P}=0.017)$ in patients with TBAD than in healthy controls (Table 2). The diameters of the sinotubular junction level ( $3.88 \pm 0.50$ vs. $3.22 \pm 0.60 \mathrm{~cm}, \mathrm{P}<0.001$ ), ascending aorta ( $3.33 \pm 0.44$ vs. $3.69 \pm 0.38 \mathrm{~cm}, \mathrm{P}<0.001)$, aortic arch (3.25 \pm 0.74 vs. $2.61 \pm 0.36 \mathrm{~cm}, \mathrm{P}<0.001)$, and descending aorta $(3.57 \pm 0.82$ vs. $2.44 \pm 0.39 \mathrm{~cm}, \mathrm{P}<0.001)$ were larger in TBAD patients than in healthy controls (Table 3). The width $(80.04 \pm 17.27$ vs. $71.73 \pm 15.55 \mathrm{~cm}, \mathrm{P}=0.005)$ and height $(24.92 \pm 11.39$ vs. $19.37 \pm 10.10 \mathrm{~cm}, \mathrm{P}<0.001)$ of the aortic arch in patients with TBAD were both larger than those of healthy controls (Table 4). There were no 
Table 2 The comparison of aortic length between the TBAD group and Healthy controls group

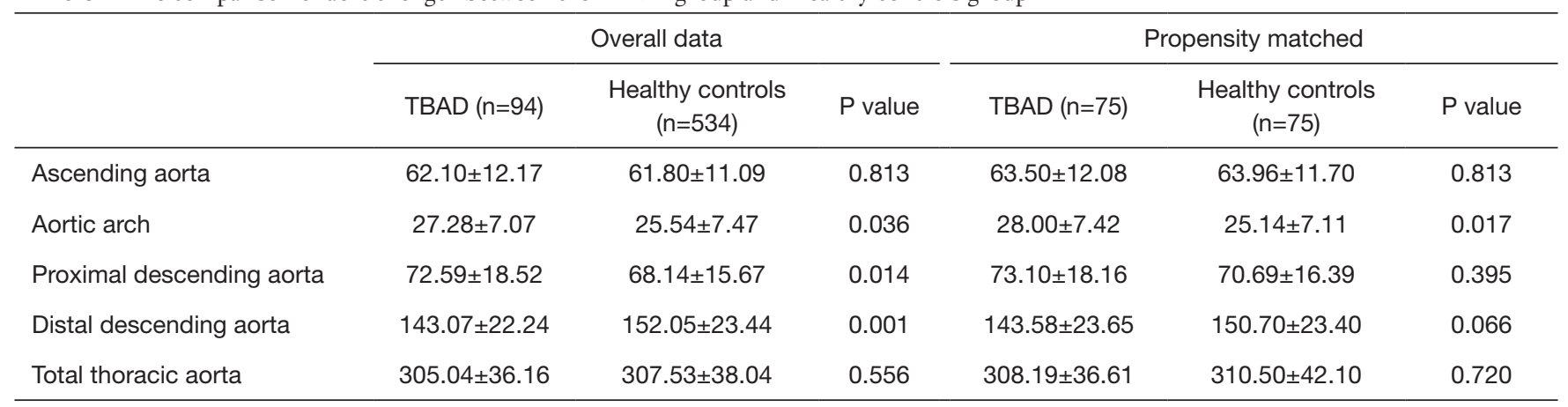

TBAD, type B aortic dissection.

Table 3 The comparison of aortic diameter between the TBAD group and Healthy controls group

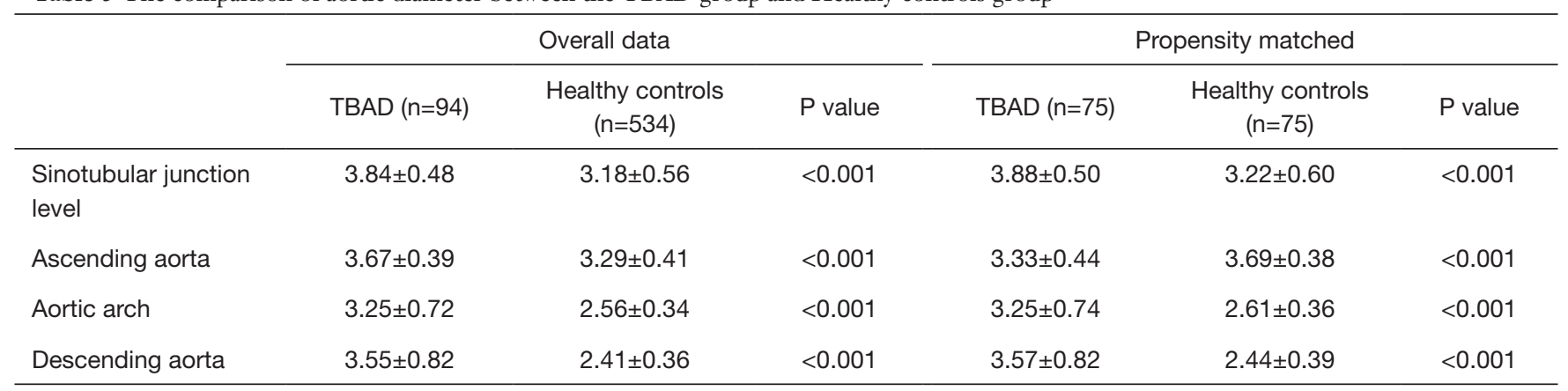

TBAD, type B aortic dissection.

Table 4 The comparison of aortic arch geometry between the TBAD group and Healthy controls group

\begin{tabular}{|c|c|c|c|c|c|c|}
\hline & \multicolumn{3}{|c|}{ Overall data } & \multicolumn{3}{|c|}{ Propensity matched } \\
\hline Aortic arch height, mm & $24.14 \pm 11.55$ & $18.53 \pm 8.94$ & $<0.001$ & $24.92 \pm 11.39$ & $19.37 \pm 10.10$ & $<0.001$ \\
\hline
\end{tabular}

TBAD, type B aortic dissection.

significant differences in the length of the ascending aorta $(\mathrm{P}=0.813)$, the lengths of the proximal $(\mathrm{P}=0.395)$ and distal descending aorta $(\mathrm{P}=0.066)$, and the length of the total thoracic aorta $(\mathrm{P}=0.720)$ between the two matched groups. There were no significant differences in arch angle $(\mathrm{P}=0.977)$ between the two matched groups.

\section{Discussion}

It is difficult to diagnose TBAD until its acute and catastrophic symptoms occur. Because of the difficulty of treating this fatal aortic disease, identification of potential risk factors before the occurrence of typical symptoms may be the most effective way to reduce the mortality associated with TBAD (2). Structural and functional changes play important roles in the process of aortic disease $(10,16)$, and one of the important changes is in the geometry of the aorta. The current guidelines recommend that the only morphological risk factor is aortic dilation, i.e., a descending aortic diameter greater than $5.5 \mathrm{~cm}$, which requires surgical 
treatment (2), but this recommendation is controversial: $60 \%$ of TBAD patients have maximum aortic diameters $<5.5 \mathrm{~cm}$, and $40 \%$ of TBAD patients had aortic diameters $<5.0 \mathrm{~cm}$ in the IRAD Registry (17). Therefore, identifying possible morphological factors other than aortic diameter may better predict the occurrence of TBAD.

Our results are similar to those of previous studies. Several previous studies have demonstrated an association between Stanford A aortic dissection and ascending aortic elongation $(7-9,12,13,16)$. Besides, other previous study has confirmed that the aortic arch tortuosity is significantly increased in patients who are at increased risk for thoracic aortic aneurysms or aortic dissections (18). As for TBAD, a study confirmed that the aortic arch elongation is associated with the development of was highly effective in predicting Stanford B dissection, exceeding the indexes such as diameter (8).

The most common site for a proximal intimal tear in TBAD is just distal to the origin of the left subclavian artery, which is the junction of the aortic arch and the proximal descending aorta (3). According to previous studies, aortic elongation with advancing age was also most pronounced in this segment $(5,7)$. Furthermore, TBAD is associated with elongation of the aortic arc (10). All of the above evidence suggests that the morphology of the aortic arch and surrounding aortic segments may be a positive marker of aortic dissection. However, previous studies have mostly focused on the diameter and length of the aorta and have not systematically explored the correlation between TBAD and the geometry of the aortic arch, such as the aortic arch width, the aortic arch height, and the inclination of the aortic arch (i.e., arch angle) $(10,19)$. In addition, the morphology of the aorta is associated with factors such as age, gender, BSA, and some comorbidities, especially hypertension, but these confounding factors were not excluded in previous studies (10-12). In this study, after analyzing 94 patients with TBAD and 543 healthy control subjects and using PSM analysis to reduce the potential for confounding by baseline factors, we revealed that TBAD was associated with longer lengths of aortic arch and with larger arch height and width.

Previous studies have confirmed that aortic arch elongation is associated with the development of TBAD (10). At the same time, elongation of the aorta with aging is most pronounced in the aortic arch $(5,7)$. The reason may be attributed to the following: Compared with the fixed parts of the thoracic aorta, including segments connected to the pericardium and diaphragm (i.e., ascending aorta and distal descending thoracic aorta), the mobile proximal descending aorta and aortic arch can move relatively freely in the longitudinal direction (19). The proximal aorta has the greatest elastin content, which makes it the release point of the twisting thoracic aorta $(17,20)$. This age-related aortic looseness may lead to changes in the geometry of the aortic arch. The abovementioned series of aortic arch morphological changes were related to TBAD in our study. The reason may be that changes in the morphology of the aortic arch may affect the hemodynamics of the descending aorta, resulting in increased mechanical stresses $(4,5)$. Experiments have shown that this increase in wall shear stress leads to changes in endothelial cells (6), which in turn exacerbate the process of degeneration of the aorta, thereby accelerating the dilation, elongation, and tortuosity of the aortic arch and descending aorta, ultimately leading to descending aortic pathology.

Of course, the most direct method to identify the morphological risk factor of aortic dissection is to compare the geometry of the nondissected aorta with the same dissected aorta, but it is obviously difficult to obtain imaging prior to dissection onset. We used Heuts's retrospective modeling method to obtain the adjusted pre-dissection dimension $(12,14)$. They revealed that the lengths and diameters of the ascending aorta and the aortic arch did not change after the occurrence of TBAD. However, in TBAD, the proximal aorta of left subclavian artery is not dissected, which is why its geometric changes cannot be a direct consequence of dissection. Therefore, it is meaningful to compare the arch morphology of the TBAD group and the Healthy control group.

The results of this study and those of previous studies support the hypothesis that aortic dissection is associated with age-related aortic morphological changes (5-14). The results of this study provide a direction for future research. In future studies, we expect to find an optimal predictor to predict the incidence of TBAD, which ideally should be a simple repeatable parameter to describe the shape of the aortic arch. If we can screen the high-risk population based on this indicator, we might prevent patients from developing TBAD at an early age, as morphological deterioration would not have been occurring long enough to cause aortic dissection. For patients with adverse aortic arch morphology, it may be necessary to increase the number of outpatient follow-up visits. For these patients, blood pressure should be strictly controlled with antihypertensive drugs, and other risk factors must be eliminated. However, we need more evidence to verify 
these results. In future studies, we also need to identify hemodynamic and histological risk factors with numerical and experimental evidence.

This study revealed the morphological factors related to TBAD after controlling for confounding factors, but several limitations should be considered. First, this study was a retrospective cross-sectional study, and the study was not without biases. Even though PSM was used to control for confounding factors, the bias of reverse causality could not be eliminated. Second, the population of healthy patients included in this study was from a group of patients evaluated in the radiology department due to other diseases rather than from a population of healthy volunteers. A prospective life-long cohort study involving a healthy volunteer population may provide more convincing evidence. However, due to the low incidence of aortic dissection, it should be considered that such an ideal study would actually be difficult to perform. Third, due to the limitations of the retrospective study, some risk factors, such as family history of dissection in the control group, could not be collected; therefore, these confounding factors could not be excluded from the two groups. Fourth, we measured aortic morphology in the healthy controls using non-ECG-gated CT, as differences over the cardiac cycle might affect the measurements. Fifth, the results of this study can only provide a correlation between Stanford $\mathrm{B}$ aortic dissection and the morphological changes of the aortic arch. Whether there is a causal relationship between these morphological risk factors and aortic dissection needs to be provided by prospective studies that can provide a causal link.

\section{Conclusions}

This study demonstrates that TBAD was associated with longer lengths of aortic arch and with larger arch height and width. TBAD is associated with morphological factors other than aortic diameter, especially the geometry of the aortic arch.

\section{Acknowledgments}

The authors wish to thank Meirong Xi for her important contribution to data collection.

Funding: This work was supported by Natural Science Foundation of China (81870346, 81701842, 81701801,81700432), Clinical Research Program of 9th People's Hospital, Shanghai Jiao Tong University
School of Medicine (JYLJ019) and Shanghai Jiao Tong University School of Medicine Doctoral Innovation Fund (BXJ201935).

\section{Footnote}

Reporting Checklist: The authors have completed the STROBE reporting checklist. Available at http://dx.doi.org/10.21037/ jtd-20-1643

Data Sharing Statement: Available at http://dx.doi. org/10.21037/jtd-20-1643

Conflicts of Interest: All authors have completed the ICMJE uniform disclosure form (available at http://dx.doi. org/10.21037/jtd-20-1643). The authors have no conflicts of interest to declare.

Ethical Statement: The authors are accountable for all aspects of the work in ensuring that questions related to the accuracy or integrity of any part of the work are appropriately investigated and resolved. The procedures followed were in accordance with the Declaration of Helsinki (as revised in 2013), and the study protocol was reviewed and approved by the local ethics committee (SH9H-2019-T144-2). The need for written patient consent was waived because of the observational nature of this study. This retrospective factorial study was registered with the Chinese Clinical Trial Registry, and its registration number is ChiCTR2000029219.

Open Access Statement: This is an Open Access article distributed in accordance with the Creative Commons Attribution-NonCommercial-NoDerivs 4.0 International License (CC BY-NC-ND 4.0), which permits the noncommercial replication and distribution of the article with the strict proviso that no changes or edits are made and the original work is properly cited (including links to both the formal publication through the relevant DOI and the license). See: https://creativecommons.org/licenses/by-nc-nd/4.0/.

\section{References}

1. Evangelista A, Isselbacher EM, Bossone E, et al. Insights from the international registry of acute aortic dissection: a 20-year experience of collaborative clinical research. Circulation 2018;137:1846-60.

2. Hiratzka LF, Bakris GL, Beckman JA, et al. 2010 ACCF/AHA/AATS/ACR/ASA/SCA/SCAI/SIR/STS/ 
SVM guidelines for the diagnosis and management of patients with Thoracic Aortic Disease: a report of the American College of Cardiology Foundation/American Heart Association Task Force on Practice Guidelines, American Association for Thoracic Surgery, American College of Radiology, American Stroke Association, Society of Cardiovascular Anesthesiologists, Society for Cardiovascular Angiography and Interventions, Society of Interventional Radiology, Society of Thoracic Surgeons, and Society for Vascular Medicine. Circulation 2010;121:e266-369.

3. Riambau V, Böckler D, Brunkwall J, et al. Editor's Choice - Management of Descending Thoracic Aorta Diseases: Clinical Practice Guidelines of the European Society for Vascular Surgery (ESVS). Eur J Vasc Endovasc Surg 2017;53:4-52.

4. Van Doormaal MA, Kazakidi A, Wylezinska M, et al. Hemodynamics in the mouse aortic arch computed from MRI-derived velocities at the aortic root. J R Soc Interface 2012;9:2834-44.

5. Redheuil A, Yu WC, Mousseaux E, et al. Age-related changes in aortic arch geometry: relationship with proximal aortic function and left ventricular mass and remodeling. J Am Coll Cardiol 2011;58:1262-70.

6. Mohamied Y, Sherwin SJ, Weinberg PD. Understanding the fluid mechanics behind transverse wall shear stress. J Biomech 2017;50:102-9.

7. Adriaans BP, Heuts S, Gerretsen S, et al. Aortic elongation part I: the normal aortic ageing process. Heart 2018;104:1772-7.

8. Krüger T, Forkavets O, Veseli K, et al. Ascending aortic elongation and the risk of dissection. Eur J Cardiothorac Surg 2016;50:241-7.

9. Krüger T, Oikonomou A, Schibilsky D, et al. Aortic elongation and the risk for dissection: the Tübingen Aortic Pathoanatomy (TAIPAN) project. Eur J Cardiothorac Surg 2017;51:1119-26.

10. Lescan M, Veseli K, Oikonomou A, et al. Aortic

Cite this article as: Qiu P, Liu J, Chen Y, Zha B, Ye K, Qin J, Hao P, Kang J, Zhang C, Zhu H, Lu X. Changes in aortic arch geometry and the risk for Stanford B dissection. J Thorac Dis 2020;12(12):7193-7201. doi: 10.21037/jtd-20-1643
Elongation and Stanford B Dissection: The Tübingen Aortic Pathoanatomy (TAIPAN) Project. Eur J Vasc Endovasc Surg 2017;54:164-9.

11. Poullis MP, Warwick R, Oo A, et al. Ascending aortic curvature as an independent risk factor for type A dissection, and ascending aortic aneurysm formation: a mathematical model. Eur J Cardiothorac Surg 2008;33:995-1001.

12. Heuts S, Adriaans BP, Gerretsen S, et al. Aortic elongation part II: the risk of acute type A aortic dissection. Heart 2018;104:1778-82.

13. Wu J, Zafar MA, Li Y, Saeyeldin A, et al. Ascending aortic length and risk of aortic adverse events: the neglected dimension. J Am Coll Cardiol 2019;74:1883-94.

14. Rylski B, Muñoz C, Beyersdorf F, et al. How does descending aorta geometry change when it dissects? Eur J Cardiothorac Surg 2018;53:815-21.

15. Benedetto U, Head SJ, Angelini GD, et al. Statistical primer: propensity score matching and its alternatives. Eur J Cardiothorac Surg 2018;53:1112-7.

16. Akin I, Nienaber CA. Age-dependent aortic elongation: a new predictor for type A aortic dissection? Heart 2018;104:1729-30.

17. Ohyama Y, Redheuil A, Kachenoura N, et al. Imaging Insights on the Aorta in Aging. Circ Cardiovasc Imaging 2018;11:e005617.

18. Alhafez BA, Ocazionez D, Sohrabi S, Sandhu H, Estrera A, Safi HJ, Evangelista A, Hurtado LD, Guala A, Prakash SK. Aortic arch tortuosity, a novel biomarker for thoracic aortic disease, is increased in adults with bicuspid aortic valve. Int J Cardiol 2019;284:84-9.

19. Elefteriades JA. Natural history of thoracic aortic aneurysms: indications for surgery, and surgical versus nonsurgical risks. Ann Thorac Surg 2002;74:S1877-80; discussion S1892-8.

20. Tsamis A, Krawiec JT, Vorp DA. Elastin and collagen fibre microstructure of the human aorta in ageing and disease: a review. J R Soc Interface 2013;10:20121004. 